\title{
Learning Curve for Robot-Assisted Percutaneous Pedicle Screw Placement in Thoracolumbar Surgery
}

\author{
Jeremy K. T. Kam ${ }^{1}$, Calvin Gan ${ }^{2}$, Stefan Dimou ${ }^{1}$, Mohammed Awad ${ }^{1}$, \\ Bhadu Kavar ${ }^{1}$, Girish Nair ${ }^{1}$, Andrew Morokoff ${ }^{1}$ \\ ${ }^{1}$ Department of Neurosurgery, Royal Melbourne Hospital, Parkville, VIC, Australia \\ ${ }^{2}$ Department of Radiology, Royal Melbourne Hospital, Parkville, VIC, Australia
}

Study Design: Retrospective review of an initial cohort of consecutive patients undergoing robot-assisted pedicle screw placement. Purpose: We aimed to evaluate the learning curve, if any, of this new technology over the course of our experience.

Overview of Literature: Percutaneous pedicle screws have specific advantages over open freehand screws. However, they require intraoperative imaging for their placement (e.g., fluoroscopy and navigation) and require increased surgeon training and skill with the learning curve estimated at approximately 20-30 cases. To our knowledge, this is the first study that measures the learning curve of robot-guided purely percutaneous pedicle screw placement with comprehensive objective postoperative computed tomography (CT) scoring, time per screw placement, and fluoroscopy time.

Methods: We included the first 80 consecutive patients undergoing robot-assisted spinal surgery at Melbourne Private Hospital. Data were collected for pedicle screw placement accuracy, placement time, fluoroscopy time, and revision rate. Patient demographic and relevant perioperative and procedural data were also collected. The patients were divided equally into four sub-groups as per their chronological date of surgery to evaluate how the learning curve affected screw placement outcomes.

Results: Total 80 patients were included; $73(91 \%)$ had complete data and postoperative CT imaging that could help assess that placement of 352 thoracolumbar pedicle screws. The rate of clinically acceptable screw placement was high $(96.6 \%, 95.4 \%, 95.6 \%$, and $90.7 \%$, in groups 1 to 4 , respectively, $p=0.314)$ over time. The median time per screw was 7.0 minutes $(6.5,7.0,6.0$, and 6.0 minutes in groups 1 to 4 , respectively, $p=0.605)$. Intraoperative revision occurred in only 1 of the 352 screws $(0.3 \%)$.

Conclusions: We found that robot-assisted screw placement had high accuracy, low placement time, low fluoroscopy time, and a low complication rate. However, there were no significant differences in these parameters at the initial experience and the practiced, experience placement (after approximately 1 year), indicating that robot-assisted pedicle screw placement has a very short (almost no) learning curve.

Keywords: Robotic surgical procedures; Pedicle screws; Spinal fusion

\section{Introduction}

Percutaneous pedicle screws have become the standard of care in spinal fusion surgery because of their multiple potential advantages, including more lateral placement, avoidance of the superior facet joint, stronger biome-

\footnotetext{
Received Jan 28, 2019; Revised Feb 28, 2019; Accepted Mar 16, 2019

Corresponding author: Jeremy K. T. Kam

Department of Neurosurgery, The Royal Melbourne Hospital, Grattan Street, Parkville Vic 3050, Australia

Tel: +61-3-9342 8219, Fax: +61-3-9342 7273, E-mail: drjeremykam@gmail.com
} 
chanical pull out strength, smaller incisions, less bleeding, lower pain, less infection, and possibly better long-term outcomes [1-8]. However, they depend on some form of intraoperative imaging for placement (e.g., fluoroscopy and computer guided image navigation) and require highlevel surgeon training and skill, with the learning curve being completed, in terms of operative time and complications, once the surgeon has performed about 20-30 screw placements $[9,10]$.

Robot-assisted pedicle screw placement ensures anatomical orientation and mechanical control of the instrumentation (placement of the cannula, drill position and trajectory, and placement of the pedicle screw). This provides additional advantages over navigated or fluoroscopy-guided pedicle screw placement by reducing the $6^{\circ}$ of motion in a three-dimensional space of Jamshidi needle placement to $2^{\circ}$ of freedom (depth of tool insertion and tool clockwise/counter-clockwise rotation). Moreover, it only uses 2 shots of bi-planar radiography to register to the preoperative computed tomography (CT); therefore, the Renaissance robot can significantly save fluoroscopy time and staff radiation exposure.

This is a relatively new technology; therefore, limited studies have been conducted on the accuracy and advantages of Renaissance robot. Currently, there is growing evidence that robot-guided screws are more accurate than conventional methods of percutaneous pedicle screw placement with most retrospective studies and trials reporting nearly $99 \%$ accuracy of clinically acceptable screw positioning [11-16].

We aimed to evaluate the learning curve, if any, of this new technology by measuring the screw placement time and accuracy, fluoroscopy exposure, revision rate, and complications over the course of our experience. To our knowledge, this is the first study to assess the learning curve of robot-guided purely percutaneous pedicle screw placement with comprehensive objective postoperative CT scoring, time per screw, and fluoroscopy time.

\section{Materials and Methods}

\section{Study setting and participants}

Between February 2016 and May 2017, when robotassisted pedicle screw placement was first instituted, 80 consecutive patients for whom spinal surgery was performed by the three senior authors (AM, BK, and GN) were included. The surgeries were performed for several indications; the most common indication being degenerative disk disease, at Melbourne Private Hospital. All the surgeons have a subspecialty spinal practice as neurosurgeons. The results were obtained from retrospective records and perioperative imaging review. All the cases involved percutaneous pedicle screw placement. Individual pedicle screw placement accuracy, time, fluoroscopy time, and revision rate were recorded. Patient demographic and relevant data, such as surgical indication, comorbidities, number of spinal levels undergoing surgery, and complications were recorded. Human Research Ethics Committee (HREC 2017.202) approval was obtained for the study, and written consent was not required for retrospective data review. All patients undergoing our initial robotassisted thoracolumbar surgery experience were included; therefore, there were no specific exclusion criteria. The patients were divided equally into four sub-groups as per their chronological date of surgery to evaluate how the learning curve affected screw placement accuracy, fluoroscopy time, and screw placement time. Patients were excluded if incomplete data were available.

\section{Outcomes}

For each case, screw placement accuracy, screw placement time, fluoroscopy time, and revision rate were measured prospectively. Each screw was scored independently by two authors (JK and CG) using the Gertzbein-Robbins classification [17] (Fig. 1) based on the postoperative CT images obtained for all patients $[18,19]$. Any discrepancy was resolved by consensus and review. Complications and adverse events were also reviewed. The outcomes were compared between the groups.

\section{Surgical technique}

All the patients underwent percutaneous screw placement using The Renaissance Guidance System (Mazor Robotics Ltd., Caesarea, Israel; 'Mazor robot'), a navigation device used in spinal surgery for the placement of screws into the spine. The technique involves matching an on-table bi-planar fluoroscopic image to a preoperative $\mathrm{CT}$ of the spine, wherein pre-planned screws have been designed on a computer by the surgeon. A mechanical robot arm attached to the table and/or patient then moves to place a guide tube that assists the surgeon to drill a pilot hole and 
(A)

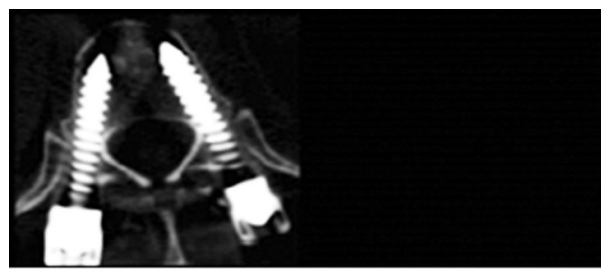

$(0 \mathrm{~mm})$

(B)

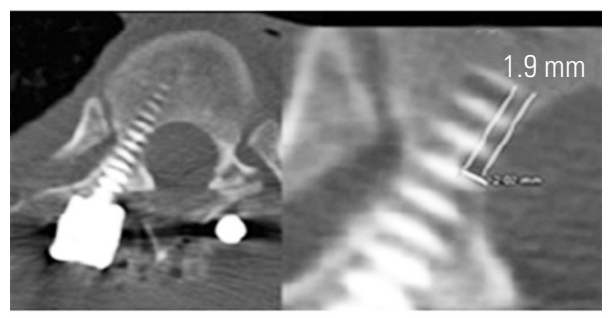

$(>2 \mathrm{~mm})$

(C)

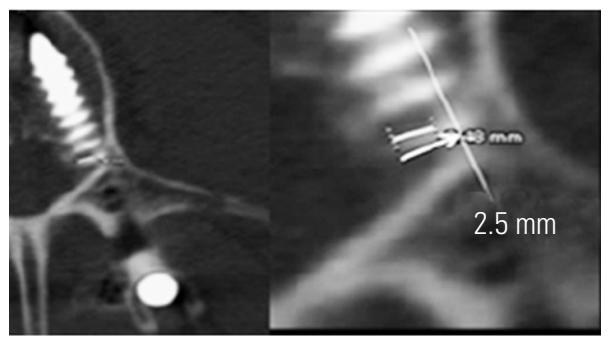

$(>4 \mathrm{~mm})$

(D)

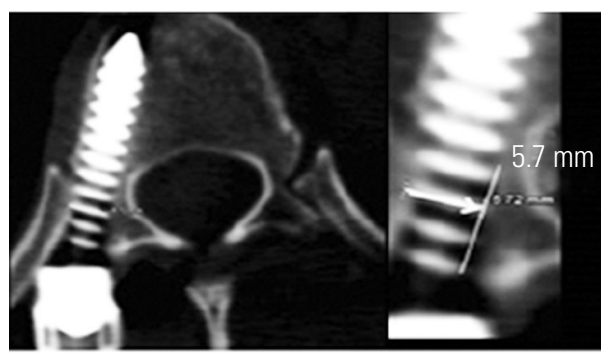

$(>6 \mathrm{~mm})$

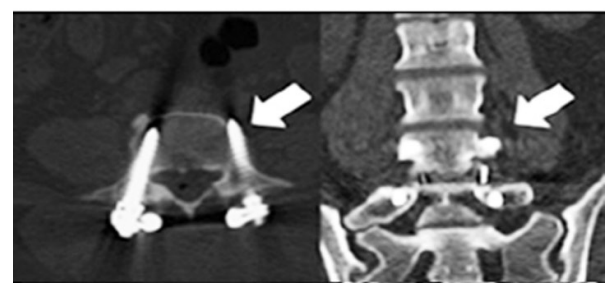

$(\geq 6 \mathrm{~mm})$

\section{(E)}

Fig. 1. Computed tomography scans demonstrating the GertzbeinRobbins classification of screw positioning. The grades reflect the deviation of the screw from the 'ideal' intrapedicular trajectory. (A) Grade $A$ is an intrapedicular screw without breach of the cortical layer of the pedicle; $(\mathbf{B})$ grade $B$ reflects a screw that breaches the cortical layer of the pedicle; however, it does not exceed it laterally by $>2$ $\mathrm{mm}$. (C, D) Grades $C$ and D indicate a penetration of $<4$ and $<6 \mathrm{~mm}$, respectively. (E) Grade $\mathrm{E}$ indicates a screw that does not pass through the pedicle or that, at any given point in its intended intrapedicular course, breaches the cortical layer of the pedicle in any direction by $\geq 6 \mathrm{~mm}$ (arrow). Reproduced from Schatlo et al. J Neurosurg Spine 2014;20:636-43, with permission from American Association of Neurological Surgeons [17].

accurately place a guide wire over which a spinal screw can be inserted.

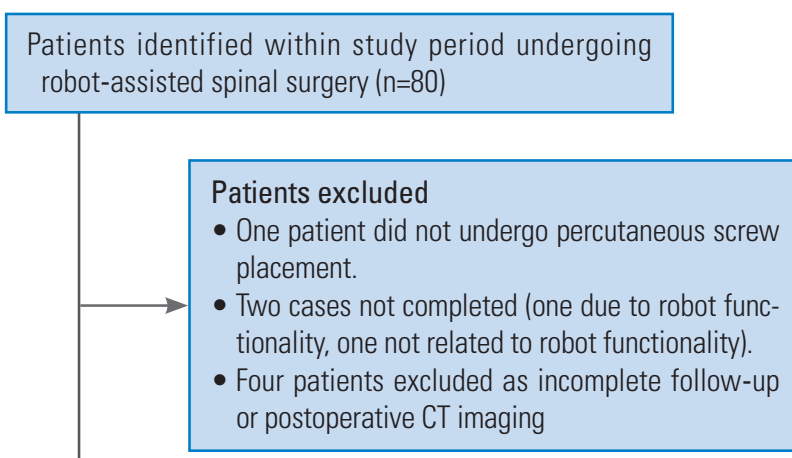

Patients with completed follow-up and CT imaging $(n=73)$

Fig. 2. Patient flow diagram. CT, computed tomography.

\section{Statistical analyses}

Group comparisons were conducted using Fisher's exact, chi-square, and Freeman-Halton extension tests with a significance level of $p<0.05$. Continuous variables were compared using the Mann-Whitney $U$, Kruskal-Wallis, and one way analysis of variance tests, as appropriate. IBM SPSS ver. 20.0 (IBM Corp., Armonk, NY, USA) was used to perform the statistical analyses and generate graphs.

\section{Results}

\section{Patients}

Total 80 patients were admitted to undergo robot-assisted spinal surgery for several indications within the study period (Fig. 2). Baseline characteristics of the groups are described in Table 1. Complete data on demographic profile, presentation details, complications, and postoperative CT imaging to assess hardware placement were available for 73 patients (91\%). The mean \pm standard deviation age of the study participants was $66.6 \pm 11.1$ years, and $63.0 \%$ were women. There were no significant differences between the groups for comorbidities, number of levels operated, indication, or approach. Significantly higher numbers of patients underwent thoracic surgery in the third quarter of the group's experience (Table 1).

\section{Primary outcomes}

Total 352 thoracolumbar percutaneous pedicle screws were placed for 73 patients; of these, $90.1 \%$ were placed in an ideal position (grade A) without evidence of breach. 
Table 1. Demographic and clinical characteristics of study participants

\begin{tabular}{|c|c|c|c|c|c|c|}
\hline Characteristic & Total $(n=73)$ & Group $1(n=18)$ & Group 2 (n=18) & Group $3(n=18)$ & Group 4 (n=19) & $p$-value ${ }^{\text {a) }}$ \\
\hline \multicolumn{7}{|l|}{ Patient demographics } \\
\hline Age (yr) & $66.6 \pm 11.1$ & $69.3 \pm 8.1$ & $65.6 \pm 8.1$ & $67.3 \pm 11.8$ & $64.2 \pm 14.9$ & 0.549 \\
\hline Female gender & $46(63.0)$ & $12(66.7)$ & $13(72.2)$ & $12(66.7)$ & $9(47.4)$ & 0.414 \\
\hline \multicolumn{7}{|l|}{ Patient characteristics } \\
\hline Diabetes & $8(11.0)$ & $1(5.6)$ & $2(11.1)$ & $3(16.7)$ & $2(10.5)$ & 0.863 \\
\hline Cardiovascular disease & $18(24.7)$ & $7(38.9)$ & $2(11.1)$ & $4(22.2)$ & $5(26.3)$ & 0.279 \\
\hline Pulmonary disease & $5(6.8)$ & $1(5.6)$ & $1(5.6)$ & $2(11.1)$ & $1(5.3)$ & 0.934 \\
\hline Osteoporosis & $5(6.8)$ & 0 & $2(11.1)$ & $1(5.6)$ & $2(10.5)$ & 0.745 \\
\hline Steroid use & $5(6.8)$ & $2(11.1)$ & $2(11.1)$ & $1(5.6)$ & 0 & 0.469 \\
\hline \multicolumn{7}{|l|}{ Surgical characteristics } \\
\hline Degenerative & $63(86.3)$ & $16(88.9)$ & $16(88.9)$ & $13(72.2)$ & $18(94.7)$ & \\
\hline Neoplastic & $6(8.2)$ & $1(5.6)$ & $1(5.6)$ & $3(16.7)$ & $1(5.3)$ & 0.588 \\
\hline Trauma/fracture & $4(5.5)$ & $1(5.6)$ & $1(5.6)$ & $2(11.1)$ & $0(0.0)$ & 0.360 \\
\hline Single level/multilevel & $53(72.6) / 20(27.4)$ & $11(61.1) / 7(38.9)$ & $14(77.8) / 4(22.2)$ & $12(66.7) / 6(33.3)$ & $16(84.2) / 3(15.8)$ & 0.407 \\
\hline Thoracic/lumbar & 8 (11.0) /65 (89.0) & $1(5.6) / 17(94.4)$ & $2(11.1) / 16(88.9)$ & $5(27.8) / 13(72.2)$ & $0 / 19(100.0)$ & $0.032^{*}$ \\
\hline Latera/XLIF & $36(49.3)$ & $12(66.7)$ & $8(44.4)$ & $6(33.3)$ & $10(52.6)$ & \\
\hline Posterior/PLIF & $21(28.8)$ & $4(22.2)$ & $7(38.9)$ & $5(27.8)$ & $5(26.3)$ & 0.588 \\
\hline Anterior/ALIF/OLIF & $12(16.4)$ & $1(5.6)$ & $2(11.1)$ & $5(27.8)$ & $4(21.0)$ & 0.194 \\
\hline Pedicle screws only & $4(5.5)$ & $1(5.6)$ & $1(5.6)$ & $2(11.1)$ & 0 & 0.411 \\
\hline
\end{tabular}

Values are presented as mean \pm standard deviation or number (\%).

XLIF, extreme lateral interbody fusion; PLIF, posterior lumbar interbody fusion; ALIF, anterior lumbar interbody fusion; OLIF, oblique lumbar interbody fusion.

alFrom Fisher's exact, with Freeman-Halton extension, chi-square, Mann-Whitney U-test, Kruskal-Wallis, one way analysis of variance as appropriate. ${ }^{*} p<0.05$ (significant)

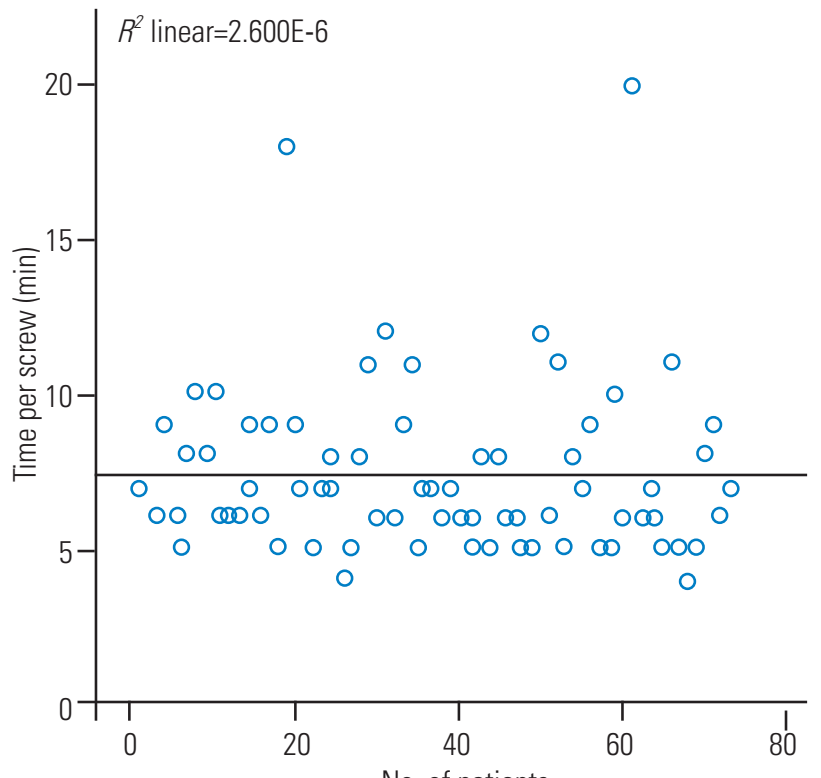

No. of patients

Fig. 3. Scatterplot of the time per screw in minutes with increasing experience: maintenance of low time per screw from the beginning throughout the surgical experience.
Distributions of ideal versus non-ideal placement of pedicle screws are given in Table 2. The rate of ideal screw placement remained unchanged among the groups. The average (median) time for screw placement was 7 minutes and did not vary significantly among the groups. Fig. 3 demonstrates screw placement time over the authors' experience that remained low from the beginning of the series. The average (median) fluoroscopy time per screw was 3 seconds. This varied significantly among the groups, with group 3 (third quarter) having a significantly $(p=0.021)$ increased fluoroscopy time per screw of 4 seconds.

\section{Secondary outcomes and adverse events}

There were no misplaced screws placed within the study period. There was one intraoperative revision $(0.3 \%)$ that was not associated with complication (Table 3). Overall, there were four complications (5.5\%). One patient expe- 
Table 2. Screw accuracy, placement, and fluoroscopy time

\begin{tabular}{|c|c|c|c|c|c|c|}
\hline Variable & $\begin{array}{c}\text { Total } \\
(n=73)\end{array}$ & $\begin{array}{c}\text { Group } 1 \\
(n=18)\end{array}$ & $\begin{array}{c}\text { Group } 2 \\
(n=18)\end{array}$ & $\begin{array}{c}\text { Group } 3 \\
(n=18)\end{array}$ & $\begin{array}{c}\text { Group } 4 \\
(n=19)\end{array}$ & $p$-value ${ }^{\text {a) }}$ \\
\hline Pedicle screws placed & $352(100.0)$ & $89(100.0)$ & $87(100.0)$ & $90(100.0)$ & $86(100.0)$ & \\
\hline \multicolumn{7}{|l|}{ Ideal screw placement (\%) } \\
\hline Grade A screws & $317(90.1)$ & $85(95.5)$ & $80(92.0)$ & $77(85.6)$ & 75 (87.2) & 0.105916 \\
\hline Grade B-E screws & $35(9.9)$ & $4(4.5)$ & $7(8.0)$ & $13(14.4)$ & $11(12.8)$ & \\
\hline \multicolumn{7}{|l|}{ Clinically acceptable screw placement (\%) } \\
\hline Grade A-B screws & $333(94.6)$ & $86(96.6)$ & $83(95.4)$ & $86(95.6)$ & $78(90.7)$ & 0.3140 \\
\hline Grade C-E screws & $19(5.4)$ & $3(3.4)$ & $4(4.6)$ & $4(4.4)$ & $8(9.3)$ & \\
\hline \multicolumn{7}{|l|}{ Distribution of screw accuracy B-E (\%) } \\
\hline Grade B & $16(4.5)$ & $1(1.1)$ & $3(3.4)$ & $9(10.0)$ & $3(3.5)$ & \\
\hline Grade C & $10(2.8)$ & $2(2.2)$ & $2(2.3)$ & $1(1.1)$ & $5(5.8)$ & \\
\hline Grade D & $7(2.0)$ & $1(1.1)$ & $1(1.1)$ & $3(3.3)$ & $2(2.3)$ & \\
\hline Grade $\mathrm{E}$ & $2(0.6)$ & $0(0.0)$ & $1(1.1)$ & $0(0.0)$ & $1(1.2)$ & \\
\hline \multicolumn{7}{|l|}{ Screw placement times (interquartile range) } \\
\hline Median time per screw in minutes & $7.0(3.0)$ & $6.5(3.0)$ & $7.0(4.0)$ & $6.0(3.0)$ & $6.0(4.0)$ & 0.605 \\
\hline Median fluoroscopy time per screw in seconds & $3.0(2.0)$ & $2.0(2.0)$ & $3.0(1.0)$ & $4.0(9.0)$ & $3.0(2.0)$ & $0.021^{*}$ \\
\hline
\end{tabular}

Values are presented as number (\%), unless otherwise stated.

a) From Fisher's exact, with Freeman-Halton extension, chi-square, Mann-Whitney U-test, Kruskal-Wallis, one way analysis of variance as appropriate. ${ }^{*} p<0.05$ (significant).

Table 3. Screw misplacement, adverse events, and complications

\begin{tabular}{|c|c|c|c|c|c|c|}
\hline Variable & $\begin{array}{c}\text { Total } \\
(\mathrm{n}=73)\end{array}$ & $\begin{array}{c}\text { Group } 1 \\
(n=18)\end{array}$ & $\begin{array}{c}\text { Group } 2 \\
(n=18)\end{array}$ & $\begin{array}{c}\text { Group } 3 \\
(n=18)\end{array}$ & $\begin{array}{c}\text { Group } 4 \\
(n=19)\end{array}$ & $p$-value ${ }^{\text {a) }}$ \\
\hline Pedicle screws placed & $352(100.0)$ & $89(100.0)$ & $87(100.0)$ & $90(100.0)$ & $86(100.0)$ & \\
\hline Intraoperative screw revision & $1(0.3)$ & 0 & 0 & 0 & $1(1.2)$ & \\
\hline Misplaced screws & 0 & 0 & 0 & 0 & 0 & \\
\hline Complications (\%) & $4(5.5)$ & 0 & $1(5.6)$ & $3(16.7)$ & 0 & 0.093 \\
\hline
\end{tabular}

Values are presented as number (\%), unless otherwise stated.

alFrom Fisher's exact, with Freeman-Halton extension, chi-square, Mann-Whitney U-test, Kruskal-Wallis, one way analysis of variance as appropriate. ${ }^{*} p<0.05$ (significant).

rienced a spinal cord stroke associated with neurological deficit. One patient underwent revision surgery for an asymptomatic extruded bone graft detected on postoperative imaging. One patient had two freehand pedicle screws placed after dysfunction with the Mazor system. One patient had bowel obstruction and acute renal injury postoperatively. There were no postoperative screw revisions or neurological deficits associated with screw placement. No complications were attributed to robot-assisted pedicle screw placement.

\section{Discussion}

In this study, we found no significant difference in the screw placement accuracy, time per screw placement, and complication rate among the 4-time periods in our initial experience, indicating no significant learning curve; further, this indicates that from the early experience of this technique, acceptable outcomes are achieved. However, the fluoroscopy time increased in the third quarter of experience (median $=4$ seconds). In our series, the overall complication rate was 5.5\% although there were no complications attributed to mal-placement of robot-assisted 
pedicle screws.

'Clinically acceptable' screws in the literature are those deemed grade A or B ( $<2 \mathrm{~mm}$ deviation from the cortex) using the Gertzbein-Robbins method [19-21]. Of the 352 pedicle screws that were placed, 333 (94.6\%) were grade A or B and 19 (5.3\%) were grade C, D, or E. A more clinically relevant outcome than screw placement accuracy is screw revision. Our intraoperative revision rate was $0.3 \%$ (1 of 352) with no postoperative evaluation of the screws requiring revision. This is similar to the results from the growing body of literature that has documented high screw placement accuracy rates. Keric et al. [13] evaluated 413 patients who had Renaissance robot-guided surgery in two German centers and found a $2 \%$ misplacement rate of 3-6 $\mathrm{mm}$ and $1.1 \%$ deviation of $>6 \mathrm{~mm}$. Nine screws required revision $(0.48 \%)$. Registration failure occurred in one patient because of obesity. Most deviated screws showed lateral deviation and probably resulted from 'skiving. Twenty-four screw misplacements were potentially due to platform dislocation, and in six screw placements, the reason for the need of revision was unclear [13]. Kim et al. [12] performed an randomized controlled trial (RCT) of robot-assisted $(n=37)$ versus fluoroscopy-guided $(n=41)$ screw placement in patients undergoing posterior lumbar interbody fusion (PLIF). Although there was no difference in pedicle screw placement accuracy, robot-guided screws were less likely to breach the proximal facet joint with better convergence angles. Hyun et al. [11] reported a RCT comparing the Renaissance robot system with traditional fluoroscopy-guided pedicle screw placement in 1- or 2-level degenerative lumbar spinal fusions. Sixty patients were enrolled, and 270 screws were placed. There was 5\% screw deviation in the fluoroscopy group compared to only $2.3 \%$ in the robot group; however, the placement of all the screws in the robotic group was clinically acceptable [11]. In the same study, Hyun et al. [11] compared the first 15 patients with the latter 15 patients in the robot group and found that time per screw decreased from 5.5 to 4.0 minutes ( $p=0.023$ ). Fluoroscopy time per screw similarly decreased from 4.1 to 2.9 seconds; however, this was not statistically significant $(p=0.117)$ [11].

Reporting of the learning curve in robot-assisted pedicle screw placement is sporadic and varied with outcomes not only relating to screw placement accuracy and revision, but also time per screw, fluoroscopy time per screw, and the effect of surgeon seniority on operating times being incorporated into larger studies that assess the vi- ability of this technique.

In our study, ideal screw placement did not vary among the groups, ranging from $85.6 \%-95.5 \%(p=0.106)$, and our median time per screw was 7 minutes, ranging from $6.0-7.0$ minutes in the four groups $(p=0.605)$, indicating that there was no significant learning curve for this technique. However, the median fluoroscopy time varied, ranging from 2-4 seconds per screw among the four groups ( $p=0.021$ ). The longest fluoroscopy times were recorded in the third quarter of our experience; this may be due to the much higher number of thoracic cases $(5 / 18$, $27.8 \%)$ in that quarter compared to that in the others $(p=0.032)$. It is noteworthy that ideal screw placement was highest in the first quarter, probably because the surgeons were more cautious while executing a new technique and had assistive supervision.

Conflicting results have been reported for the learning curve of this technique in a large study by Schatlo et al. [18] that reported an initial learning curve that included screw placement in about 25 cases; this study reported that misplacement rates peaked between the 5th and 25th surgery in 12 of 13 surgeons in a study on 258 surgeries, and 1,265 pedicle screws that were placed with robotic assistance. Contrary to this, some other studies have shown no increase in the already high accuracy of early and late experience in robot-assisted pedicle screws [22-24]. Our study expands upon previous research because as per our initial experience, the number of patients undergoing multilevel fixations in both, the thoracic and lumbar spine and the total number of patients and screws was much larger than that in the study by Kim et al. [24] who reported 20 patients undergoing robot-assisted PLIFs. The screw placement accuracy rate for open robot-assisted pedicle screws in a pediatric population for adolescent idiopathic scoliosis was unchanged when the learning curve between the first 16 and final 16 patients was examined (9.6\% versus $7.4 \%>2 \mathrm{~mm}$ deviation, $p=0.573$ ) [23]. Moreover, we measured the time per screw placement, revision rate, and fluoroscopy times to show that in other aspects of pedicle screw placement, not just accuracy there is minimal learning curve.

Fluoroscopy time decreased by $30 \%$ after the first 8 of 37 cases in a trial by Kim et al. [12] that compared robotassisted PLIF and freehand pedicle screw PLIF.

Learning curve, evaluated as time per screw placement between levels of training, was evaluated by Urakov et al. [25] in their study demonstrating that the average time 
per screw placement was 4.4 minutes for residents with $<5$ years of experience and 4.02 for residents and fellows with $\geq 6$ years of training ( $p=0.61)$. This may indicate that the learning curve is short. Although a formal evaluation of the learning curve was not conducted, the same study noted a trend of decrease in the time needed for screw placement by residents with more experience [25].

Our results indicate that there is almost no learning curve associated with robot-assisted pedicle screw placement; however, our results may not be applicable to all neurosurgeons or orthopedic surgeons because all our authors have a subspecialty interest in spine surgery. One strength of our study is that our experience is reflective of a general spine practice, with 20 of the 73 patients undergoing multilevel fixation and 10 of the 73 patients undergoing surgery for either tumor or trauma. Moreover, our case numbers are moderate, with mal-placement and adverse event rates being low; therefore, the possible complications associated with the increasing use of robotassisted spinal surgery may have been under reported. Further large prospective studies are needed to assess the factors that affect robot screw placement accuracy and its use in different pathologies, such as a deformity with rotated pedicles or altered anatomy, for a complete evaluation of this technique.

\section{Conclusions}

Our study includes a comprehensive analysis of the learning curve associated with the adoption of robot-assisted pedicle screw placement as a new surgical technique. We evaluated multiple outcomes, such as screw placement accuracy, screw placement time, and complication rate, and found that robot-assisted screws were associated with high overall accuracy, low placement and fluoroscopy times, and low complication rates; however, there was no significant difference in these parameters from the initial experience to the practiced experience (after approximately 1 year), indicating that robot-assisted pedicle screw placement has a very short to almost no learning curve.

\section{Conflict of Interest}

No potential conflict of interest relevant to this article was reported.

\section{Author Contributions}

Study design and concept: AM, GN, BK, JK; data collection, analysis: JK, CG, SD, MA; and manuscript writing: $\mathrm{JK}, \mathrm{SD}$.

\section{References}

1. Babu R, Park JG, Mehta AI, et al. Comparison of superior-level facet joint violations during open and percutaneous pedicle screw placement. Neurosurgery 2012;71:962-70.

2. Chen Z, Zhao J, Xu H, Liu A, Yuan J, Wang C. Technical factors related to the incidence of adjacent superior segment facet joint violation after transpedicular instrumentation in the lumbar spine. Eur Spine J 2008; 17:1476-80.

3. Gejo R, Matsui H, Kawaguchi Y, Ishihara H, Tsuji $H$. Serial changes in trunk muscle performance after posterior lumbar surgery. Spine (Phila Pa 1976) 1999;24:1023-8.

4. Kim HJ, Chun HJ, Kang KT, et al. The biomechanical effect of pedicle screws' insertion angle and position on the superior adjacent segment in 1 segment lumbar fusion. Spine (Phila Pa 1976) 2012;37:1637-44.

5. Knox JB, Dai JM 3rd, Orchowski JR. Superior segment facet joint violation and cortical violation after minimally invasive pedicle screw placement. Spine J 2011;11:213-7.

6. Lee KH, Yue WM, Yeo W, Soeharno H, Tan SB. Clinical and radiological outcomes of open versus minimally invasive transforaminal lumbar interbody fusion. Eur Spine J 2012;21:2265-70.

7. Moshirfar A, Jenis LG, Spector LR, et al. Computed tomography evaluation of superior-segment facetjoint violation after pedicle instrumentation of the lumbar spine with a midline surgical approach. Spine (Phila Pa 1976) 2006;31:2624-9.

8. Park Y, Ha JW. Comparison of one-level posterior lumbar interbody fusion performed with a minimally invasive approach or a traditional open approach. Spine (Phila Pa 1976) 2007;32:537-4.

9. Mobbs RJ, Sivabalan P, Li J. Technique, challenges and indications for percutaneous pedicle screw fixation. J Clin Neurosci 2011;18:741-9.

10. Sclafani JA, Kim CW. Complications associated with the initial learning curve of minimally invasive spine 
surgery: a systematic review. Clin Orthop Relat Res 2014;472:1711-7.

11. Hyun SJ, Kim KJ, Jahng TA, Kim HJ. Minimally invasive robotic versus open fluoroscopic-guided spinal instrumented fusions: a randomized controlled trial. Spine (Phila Pa 1976) 2017;42:353-8.

12. Kim HJ, Jung WI, Chang BS, Lee CK, Kang KT, Yeom JS. A prospective, randomized, controlled trial of robot-assisted vs freehand pedicle screw fixation in spine surgery. Int J Med Robot 2017;13.

13. Keric N, Doenitz C, Haj A, et al. Evaluation of robotguided minimally invasive implantation of 2067 pedicle screws. Neurosurg Focus 2017;42:E11.

14. Schroder ML, Staartjes VE. Revisions for screw malposition and clinical outcomes after robot-guided lumbar fusion for spondylolisthesis. Neurosurg Focus 2017;42:E12.

15. Devito DP, Kaplan L, Dietl R, et al. Clinical acceptance and accuracy assessment of spinal implants guided with SpineAssist surgical robot: retrospective study. Spine (Phila Pa 1976) 2010;35:2109-15.

16. Hu X, Ohnmeiss DD, Lieberman IH. Robotic-assisted pedicle screw placement: lessons learned from the first 102 patients. Eur Spine J 2013;22:661-6.

17. Schatlo B, Molliqaj G, Cuvinciuc V, Kotowski M, Schaller K, Tessitore E. Safety and accuracy of robotassisted versus fluoroscopy-guided pedicle screw insertion for degenerative diseases of the lumbar spine: a matched cohort comparison. J Neurosurg Spine 2014;20:636-43.

18. Schatlo B, Martinez R, Alaid A, et al. Unskilled un- awareness and the learning curve in robotic spine surgery. Acta Neurochir (Wien) 2015;157:1819-23.

19. Gertzbein SD, Robbins SE. Accuracy of pedicular screw placement in vivo. Spine (Phila Pa 1976) 1990;15:11-4.

20. Chenin L, Capel C, Fichten A, Peltier J, Lefranc M. Evaluation of screw placement accuracy in circumferential lumbar arthrodesis using robotic assistance and intraoperative flat-panel computed tomography. World Neurosurg 2017;105:86-94.

21. Fan Y, Du JP, Liu JJ, et al. Accuracy of pedicle screw placement comparing robot-assisted technology and the free-hand with fluoroscopy-guided method in spine surgery: an updated meta-analysis. Medicine (Baltimore) 2018;97:e10970.

22. Ringel F, Stüer C, Reinke A, et al. Accuracy of robotassisted placement of lumbar and sacral pedicle screws: a prospective randomized comparison to conventional freehand screw implantation. Spine (Phila Pa 1976) 2012;37:E496-501.

23. Macke JJ, Woo R, Varich L. Accuracy of robot-assisted pedicle screw placement for adolescent idiopathic scoliosis in the pediatric population. J Robot Surg 2016;10:145-50.

24. Kim HJ, Lee SH, Chang BS, et al. Monitoring the quality of robot-assisted pedicle screw fixation in the lumbar spine by using a cumulative summation test. Spine (Phila Pa 1976) 2015;40:87-94.

25. Urakov TM, Chang KH, Burks SS, Wang MY. Initial academic experience and learning curve with robotic spine instrumentation. Neurosurg Focus 2017;42:E4. 